\title{
Acalásia idiopática corrigida por Cardiomiotomia de Heller associada a Valvuloplastia de Toupet: relato de caso
}

\author{
Idiopathic achalasia corrected by Heller Cardiomyotomy associated with Toupet \\ Valvuloplasty: case report
}
Acalasia idiopática corregida por cardiomiotomía de Heller asociada a valvuloplastia de Toupet: reporte de caso

Maysa Queiroz Maciel ${ }^{1 *}$, Roclides Castro de Lima ${ }^{1}$, Artur Serra Neto ${ }^{1}$, João Victor Carvalho da Paz ${ }^{2}$, Ana Karoline de Almeida Mendes ${ }^{2}$, Vitória Gonçalves Alves de Oliveira ${ }^{3}$.

\begin{abstract}
RESUMO
Objetivo: Descrever a apresentação, evolução e desfecho de um caso de acalasia de origem idiopática de difícil diagnóstico tratada por via laparoscópica. Detalhamentos de caso: Paciente do sexo feminino, 44 anos, com relato de dor torácica e dificuldade para deglutir há quatro anos, bem como perda ponderal importante ao longo dos anos. Foi inicialmente diagnosticada e tratada para gastrite moderada após investigação diagnóstica com endoscopia digestiva alta. Devido a persistência dos sintomas e nova investigação, a suspeição de acalasia foi aventada após endoscopia e exame radiográfico contrastado, sendo posteriormente confirmada por manometria e corrigida através de cardiomiotomia a Heller e posterior Valvuloplastia a Toupet, evoluindo sem intercorrências. Considerações finais: A acalasia esofágica é conhecida como um distúrbio motor primário do esôfago muito estudado, de ocorrência rara e de predomínio idiopático no mundo. Na maioria dos casos, a disfagia é o sintoma predominante, além da ocorrência de êmese, dor torácica e sensação de impactação do bolo alimentar, o que torna frequente a perda de peso durante a evolução da doença. A abordagem terapêutica de maior redução na morbimortalidade dos pacientes é a técnica videolaparoscópica, com menores complicações intraoperatórias, menor tempo de internação e melhor recuperação.
\end{abstract}

Palavras-chave: Acalasia esofágica, Miotomia de Heller, Manometria, Laparoscopia.

\begin{abstract}
Objective: To describe the presentation, evolution and outcome of a case of achalasia of idiopathic origin that is difficult to diagnose treated by laparoscopy. Case details: A 44-year-old female patient with a report of chest pain and difficulty swallowing for four years, as well as significant weight loss over the years. It was initially diagnosed and treated for moderate gastritis after diagnostic investigation with upper digestive endoscopy. Due to the persistence of symptoms and a new investigation, the suspicion of achalasia was raised after endoscopy and contrasted radiographic examination, later confirmed by manometry and corrected by cardiomyotomy to Heller and subsequent valvuloplasty to Toupet, evolving without complications. Final considerations: Esophageal achalasia is known as a primary esophageal motor disorder that has been studied for a long time, is rare and has an idiopathic predominance in the world. In most cases, dysphagia is the predominant symptom, in addition to the occurrence of emesis, chest pain and a feeling of impaction of the bolus, which makes weight loss frequent during the course of the disease. The therapeutic approach with the greatest reduction in patients' morbidity and mortality is the laparoscopic technique, with less intraoperative complications, shorter hospital stay and better recovery.
\end{abstract}

Keywords: Esophageal achalasia, Heller myotomy, Manometry, Laparoscopy.

${ }^{1}$ Hospital Universitário Presidente Dutra (HUUPD), São Luís - MA. *E-mail: maysaqmaciel21@gmail.com

2 Universidade CEUMA (UNICEUMA), São Luís - MA.

${ }^{3}$ Universidade Federal do Maranhão (UFMA), São Luís - MA. 


\section{RESUMEN}

Objetivo: Describir la presentación, evolución y evolución de un caso de acalasia de origen idiopático de difícil diagnóstico tratado por laparoscopia. Detalles del caso: una paciente de 44 años con un informe de dolor en el pecho y dificultad para tragar durante cuatro años, así como una pérdida de peso significativa a lo largo de los años. Inicialmente fue diagnosticada y tratada por gastritis moderada luego de una investigación diagnóstica con endoscopia digestiva alta. Ante la persistencia de la sintomatología y una nueva investigación se planteó la sospecha de acalasia tras endoscopia y examen radiográfico contrastado, posteriormente confirmado por manometría y corregido por cardiomiotomía a Heller y posterior valvuloplastia a Toupet, evolucionando sin complicaciones. Consideraciones finales: La acalasia esofágica se conoce como un trastorno motor esofágico primario que se ha estudiado durante mucho tiempo, es poco común y tiene un predominio idiopático en todo el mundo. En la mayoría de los casos, la disfagia es el síntoma predominante, además de la aparición de emesis, dolor torácico y sensación de impactación del bolo, lo que hace que la pérdida de peso sea frecuente durante el curso de la enfermedad. El abordaje terapéutico con mayor reducción de la morbimortalidad de los pacientes es la técnica laparoscópica, con menos complicaciones intraoperatorias, menor estancia hospitalaria y mejor recuperación.

Palabras clave: Acalasia del esófago, Miotomía de Heller, Manometría, Laparoscopia.

\section{INTRODUÇÃO}

A acalasia é um distúrbio motor esofágico relativamente raro, caracterizado pela ausência de relaxamento do esfíncter esofágico inferior (EEI) com a deglutição e pela ausência de peristaltismo ao longo do corpo do esôfago. Consequentemente, o trânsito alimentar é lentificado e o paciente tipicamente experimenta o sintoma típico da doença, a disfagia (ZANINOTTO G, et al., 2019).

A patologia é mais comum entre a quinta e a sexta década de vida, mas pode ocorrer em qualquer idade, sem diferença entre os sexos. É uma doença degenerativa adquirida caracterizada pela perda seletiva de neurônios inibitórios do sistema mioentérico que causa distúrbios motores do esôfago secundários à falta de relaxamento do esfíncter esofágico inferior (portanto seu nome: a: "não", calásia: "relaxamento") (RONDÓNCARVAJAL J, et al., 2020).

A disfagia é um sintoma significativo e tende a progredir com o passar do tempo. Sintomas como tosse noturna, pirose e sensação de esvaziamento incompleto também estão presentes. Há necessidade de ingestão de água concomitante a alimentação devido à impactação do bolo alimentar. $\mathrm{E}$ em casos mais avançados, a regurgitação de alimentos não digeridos é comum. A doença tende a ser insidiosa e em fases mais avançadas há regurgitação alimentar ou episódios de pneumonia recorrente por episódios de broncoaspiração (SCHLOTTMANN F, et al., 2018; SWANSTRÖM LL, 2019).

O diagnóstico da doença é sugerido pelos achados clínicos e confirmado por meio da realização de exames específicos, como a Endoscopia Digestiva Alta (EDA), esofagografia e manometria convencional esofágica (SANTOS BC, et al., 2021). A endoscopia digestiva alta geralmente é o primeiro exame realizado pelo paciente e a depender da fase da doença não são encontradas alterações estruturais significativas, 0 que não gera grande suspeita na fase inicial da doença, quando os sintomas ainda se manifestam de forma similar a outras patologias funcionais, como a doença do refluxo gastro-esofágico ou esofagite. Neste cenário o diagnóstico não é alcançado.

A realização de exames mais precisos ajuda no diagnóstico precoce, como o estudo contrastado do esôfago e a manometria esofágica (convencional e de alta resolução). As características manométricas definidas são aperistaltismo, que é obrigatório para o diagnóstico da patologia, e relaxamento incompleto do esfíncter esofágico inferior (EEI) que em alguns casos não está presente, pressão elevada do esfíncter esofágico inferior e a pressurização esofágica também pode ser encontrada (RONDÓN-CARVAJAL J, et al., 2020).

O surgimento da manometria de alta resolução e a classificação da acalasia em subtipos, de acordo com a Classificação de Chicago, possibilitou prever a resposta ao tratamento, com adequada definição prognóstica (ANDOLFI C e FISICHELLA PM, 2019). 
Assim como o avanço nos métodos diagnósticos, o tratamento da acalasia está em constante evolução, estando atualmente com várias opções de tratamento objetivando o alívio dos sintomas, principalmente a disfagia, permitindo a melhora do estado nutricional desses pacientes e da qualidade de vida. É importante lembrar que nenhuma das estratégias são curativas e não foi encontrada o melhor método comprovado. A escolha da estratégia terapêutica vai depender da idade e do grau de acometimento do órgão, este último quesito identificado objetivamente por meio de exames como a manometria. Dentre os tipos de tratamento pode-se citar o médico-farmacológico, como uso da toxina botulínica, o endoscópico (dilatação pneumática, miotomia endoscópica), o cirúrgico convencional, ou o laparoscópico, associado ao procedimento anti-refluxo (MONTIEL-ROA AJ, et al., 2020).

O presente estudo teve como objetivo descrever o caso de uma paciente diagnosticada com acalasia idiopática, em hospital de referência do estado do Maranhão, evidenciando pontos relevantes da apresentação clínica e evolução insidiosa, abordagem diagnóstica e terapêutica, com ênfase no tratamento cirúrgico realizado por via videolaparoscópica, através de técnica de Cardiomiotomia de Heller associada a Valvuloplastia de Toupet.

\section{DETALHAMENTO DO CASO}

Esta pesquisa obedece aos Critérios da Ética em Pesquisa com Seres Humanos conforme Resolução no. 466/12 do Conselho Nacional de Saúde, dessa forma, todas as informações coletadas neste estudo são estritamente confidenciais. Como forma de garantir que o estudo segue os critérios supracitados foi realizada a assinatura do Termo de Consentimento Livre e Esclarecido, no qual a paciente autorizava o uso das informações do relato de forma científica. Somente os pesquisadores possuem conhecimento dos dados e foi garantido $o$ anonimato.

Feminino, 44 anos, natural do interior do Maranhão, foi admitida com queixa de dor torácica e dificuldade para deglutir há quatro anos. A paciente relata que desde 2016 iniciou quadro de dor retroesternal, tipo facada, de leve intensidade, sem irradiação, desencadeada pela alimentação e associada a plenitude pós-prandial, bem como sensação de impactação do bolo alimentar no esôfago. Relata ainda melhora do quadro com a ingesta de água durante as refeições e piora com alimentação copiosa. O quadro se acompanhava também de dor epigástrica, de leve intensidade, sem irradiação, que melhorava com uso de procinéticos e inibidores da bomba de prótons.

O conjunto de sintomas motivou a realização de EDA no ano de 2018, na qual se constatou o achado de gastrite moderada, com teste da urease positivo, sendo realizado tratamento com pyloripac, segundo relato. Houve melhora discreta dos sintomas, com persistência da dor restroesternal. Nova EDA de controle foi realizada 6 meses após o tratamento, onde foi identificada resistência bacteriana ao tratamento instituído, sendo então realizado novo tratamento, o qual a paciente não soube relatar as medicações utilizadas.

Há dois anos, a paciente evoluiu com piora significativa dos sintomas, com dor retroesternal de intensidade moderada a grave, tipo facada, que irradiava para dorso, disfagia para alimentos sólidos, com ingesta alimentar em quantidade cada vez menor, com preferência para alimentos pastosos, além da necessidade de indução de vômito após as principais refeições para alívio sintomático. Relata ainda perda de $10 \mathrm{~kg}$ neste período.

Há um ano, a paciente relata que iniciou quadro de regurgitação alimentar durante o sono, no qual por muitas vezes despertava com grande quantidade de alimento em cavidade oral, seguida de engasgo e broncoaspiração, com tosse e dificuldade respiratória. O agravamento dos sintomas, causando grande constrangimento em eventos sociais, levou a reclusão social da mesma, o que motivou uma nova investigação para elucidação do caso.

Em 2019, realizou nova EDA, na qual foi evidenciada grande dificuldade da progressão do endoscópio, o que levou a suspeita de acalasia, sendo referenciada ao ambulatório de cirurgia geral de centro de referência estadual no Maranhão. A paciente se apresentava com baixo peso e ao exame físico não se houve alterações dignas de nota. Realizado exame sorológico para Doença de Chagas, cujos anticorpos foram negativos. Ainda 
em 2019, foi realizado esofagograma constrastado, com achado de redução significativa da porção terminal do esôfago, aspecto sugestivo de Acalasia grau 2 (Figura 1). Na manometria esofágica foram encontradas alterações compatíveis com Acalasia vigorosa (Tabela 1).

$\mathrm{Na}$ esofagografia (Figura 1) foi observada imagem típica do distúrbio motor esofágico que resulta em dilatação do esôfago mais proximal e afilamento da porção mais distal (que foi representado pela seta), configurando assim imagem tipicamente conhecida como "bico de pássaro". Além disso, a ausência de bolha gástrica é um achado bem frequente. Com este exame também podemos excluir causas mecânicas, como obstrução por neoplasia, que simula os mesmos sintomas.

Figura 1 - Esofagografia evidenciando redução importante da porção terminal do esôfago, compatível com acalasia.

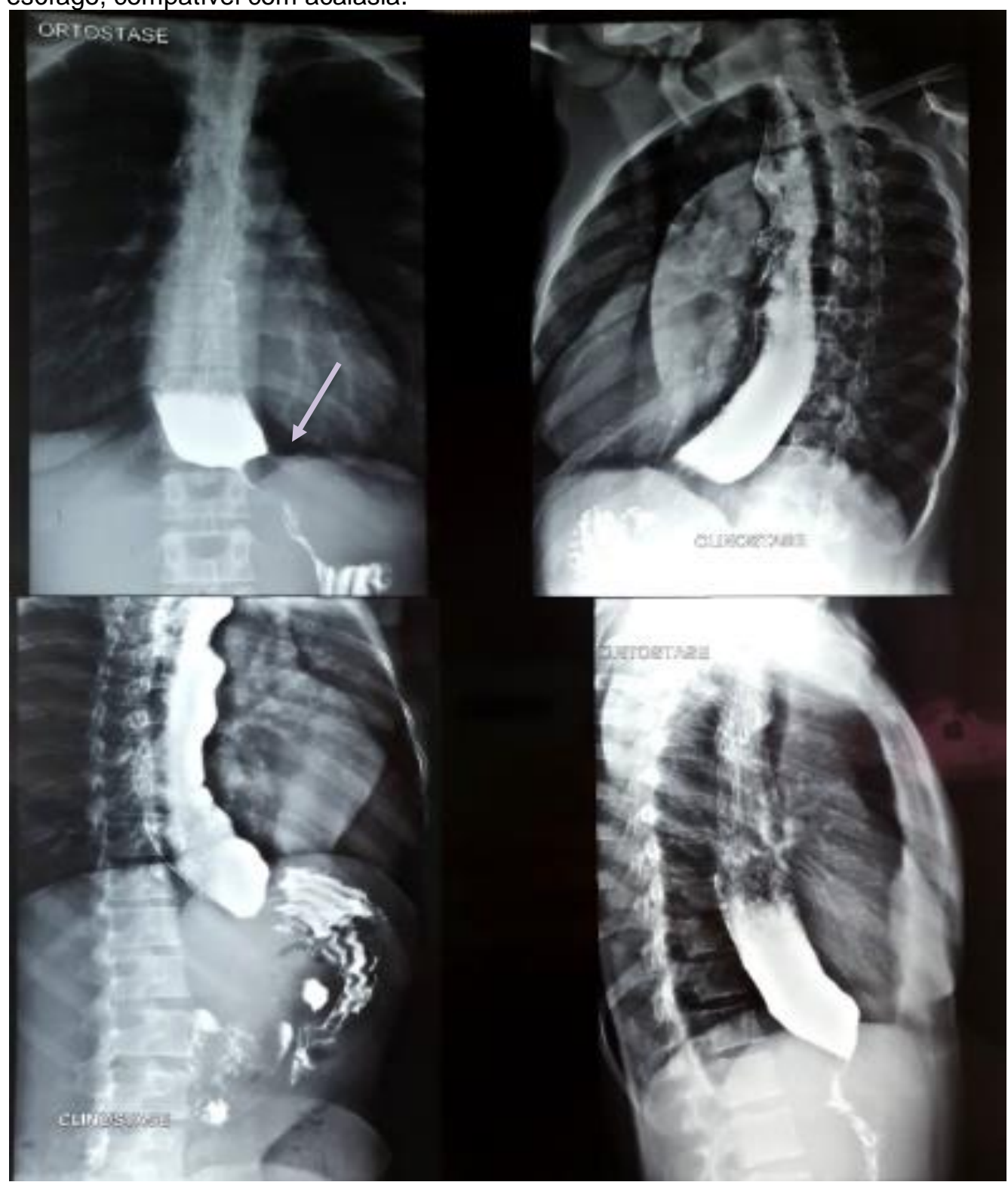

Fonte: Maciel MQ, et al., 2021.

No exame de manometria convencional realizado (Figura 2), há um esquema gráfico evidenciando as ondas em espelho no corpo esofágico (setas) a medida que as deglutições são executadas pela paciente, e são observadas em pacientes que possuem um corpo esofágico atônico. No caso da paciente, o exame evidencia ondas idênticas em todas as deglutições, não sendo evidenciada nenhuma contração eficaz durante esse período, o que ocasiona a estase alimentar no corpo esofágico. 
Figura 2 - Exame de manometria, esquema gráfico evidenciando as ondas em espelho no corpo esofágico.

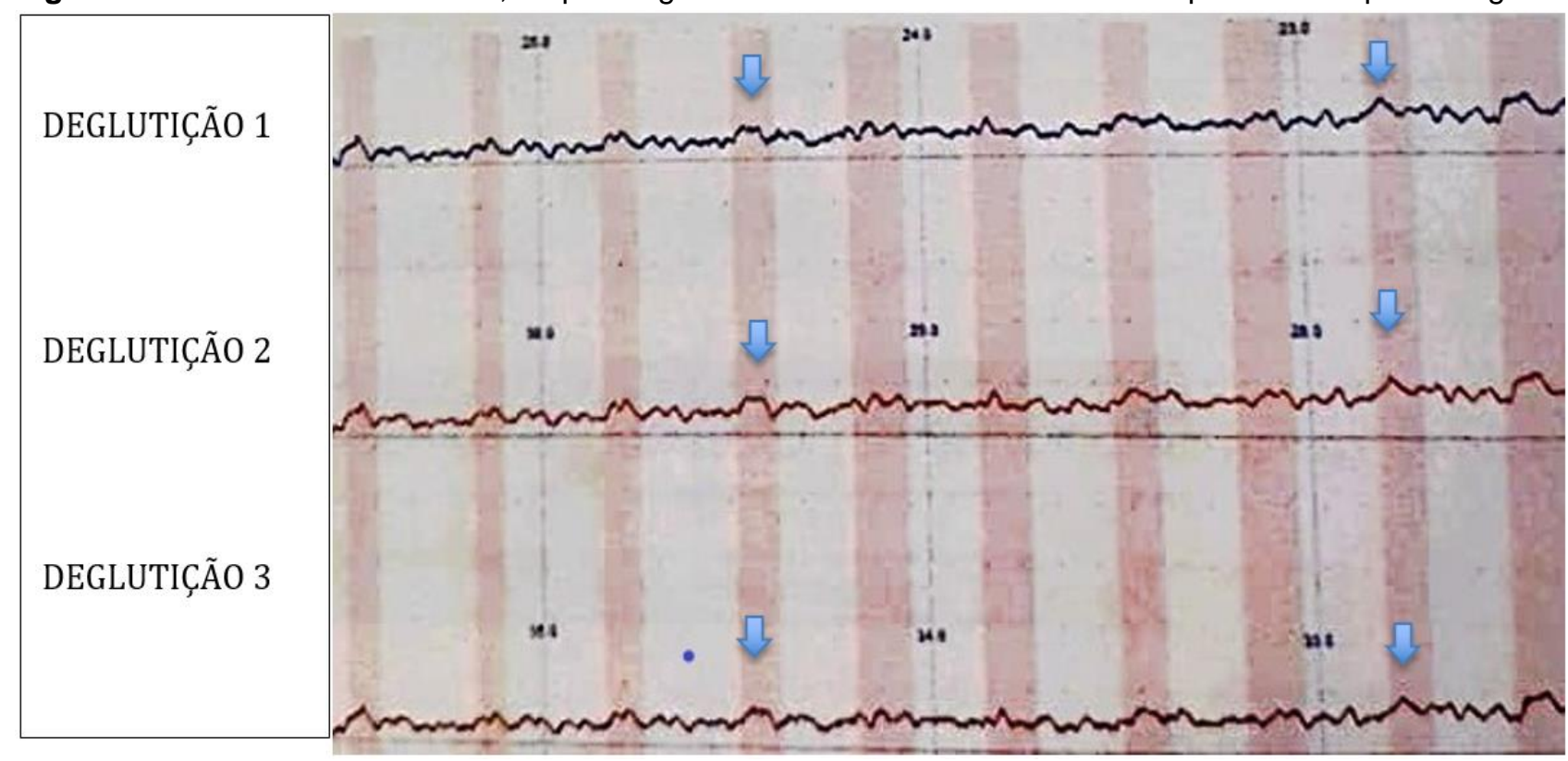

Fonte: Maciel MQ, et al., 2021.

No exame de manometria perfusional, foi ilustrada uma tabela esquemática que resume quantitativamente os dados obtidos com a manometria convencional. Percebe-se que a paciente em questão apresenta relaxamento do esfincter esofágico inferior com as deglutições, mas em contrapartida o corpo esofágico não contrai em nenhum momento, traduzindo em velocidade zero constatada com as deglutições (destaque em vermelho), o alimento permanece impactado, causando disfagia. Desta forma, a acalásia é caracterizada neste caso predominantemente pela atonia do corpo esofágico do que por ausência completa de relaxamento do esfincter esofágico.

Tabela 3 - Resumo da manometria perfusional.

\begin{tabular}{|c|c|c|c|c|c|c|c|}
\hline \multicolumn{8}{|c|}{ Esfíncter inferior do Esôfago } \\
\hline \multicolumn{8}{|c|}{ Relaxamento } \\
\hline & DEG 1 & DEG 2 & DEG 3 & DEG4 & DEG 5 & DEG 6 & Estat \\
\hline Relaxamento & Sim & Sim & Sim & Sim & Sim & Sim & $6 / 6$ \\
\hline Duração & 9"05"” & $8 " 15 " \prime$ & 7"95"' & $8 " 15 " \prime$ & 8"05"' & 8"05"' & 8"20"' \\
\hline Pressão Residual & $-2,6 \mathrm{mmHg}$ & $-0,4 \mathrm{mmHg}$ & $1,2 \mathrm{mmHg}$ & $11,5 \mathrm{mmHg}$ & $8,7 \mathrm{mmHg}$ & $1,0 \mathrm{mmHg}$ & $3,2 \mathrm{mmHg}$ \\
\hline Coordenação & Sim & Sim & Sim & Sim & Sim & Sim & $6 / 6$ \\
\hline \multicolumn{8}{|c|}{ Corpo esofágico } \\
\hline \multicolumn{8}{|c|}{ Deglutição } \\
\hline & & & \multicolumn{2}{|c|}{ DEG } & DEG & \multicolumn{2}{|r|}{ MÉDIA } \\
\hline Conduzida & & & \multicolumn{2}{|c|}{ Sim } & Sim & \multicolumn{2}{|r|}{0} \\
\hline Amplitude Média & & & & & & \multicolumn{2}{|r|}{0} \\
\hline Velocidade Distal & & & \multicolumn{2}{|c|}{0} & 0 & & 0 \\
\hline
\end{tabular}

Legenda: DEG: Deglutição; Exemplo: DEG1- primeira deglutição, DEG2 - segunda deglutição e assim sucessivamente; ESTAT: Estatística.

Fonte: Maciel MQ, et al., 2021.

A paciente foi então internada para realização de tratamento cirúrgico por videolaparoscopia, com utilização da técnica de Cardiomiotomia de Heller com posterior Valvuloplastia de Toupet, o qual se procedeu sem intercorrências ou grandes perdas sanguíneas. O pós-operatório ocorreu sem complicações, sendo a mesma liberada três dias após o procedimento. A paciente então retornou para acompanhamento 14 dias após, sem queixas, apresentando evolução favorável. 


\section{DISCUSSÃO}

A acalasia é a mais conhecida desordem motora do esôfago, sendo de origem indefinida na grande maioria dos casos. A manifestação clínica chave é a disfagia, com sensação de impactação do bolo alimentar no esôfago acompanhada de dor torácica. Esta manifestação leva muitas vezes o paciente a adquirir manobras como a ingestão de água durante as refeições aliviando a sensação de entalo. Com a evolução da patologia ocorre dilatação do esôfago com acúmulo de alimentos no órgão muitas vezes sugerida clinicamente com regurgitação de alimentos principalmente no período noturno (MONTIEL-ROA AJ, et al., 2020). No caso estudado, a paciente apresentou um quadro clássico da doença, com disfagia e sinais de doença em estágios mais avançados com regurgitação de alimentos durante o sono, tendo evoluído de forma insidiosa resultando em perda de peso expressiva no período transcorrido, além de grande limitação social.

A doença é suspeitada frente aos achados clínicos, porém o diagnóstico de certeza é feito através de exames específicos, uma vez que suas manifestações se assemelham a outras patologias do aparelho digestivo, como a doença do refluxo gastroesofágico (NETO RML, 2018). A EDA pode evidenciar retenção de alimentos, líquidos e saliva no esôfago, porém é um exame de baixa acurácia para o diagnóstico precoce (LOPES GA, 2020). Em fases mais avançadas da doença há dificuldade na realização da EDA devido estreitamento da porção distal do esôfago, como evidenciado no caso clínico em questão.

O estudo radiológico contrastado, esofagograma, é exame indispensável na definição da morfologia do órgão, com achados bastante sugestivos da doença, e com melhor sensibilidade se comparado com a endoscopia. Nele também é possível o achado de outras patologia que fazem diagnóstico diferencial com acalasia (SANTOS BC, et al., 2021). No exame, evidenciamos um aspecto clássico da doença, com afunilamento do esôfago distal, em configuração de "bico de pássaro", bem como dilatação proximal (NETO RML, 2018).

A manometria esofágica, convencional ou de alta resolução, é o exame realizado que permite diagnóstico com elevado grau de certeza mesmo em casos iniciais da doença. Atualmente, com a mamometria esofágica de alta resolução se alcança mensurações mais precisas das pressões circunferenciais intra-luminais do órgão, e com a classificação de Chicago a demonstração dos achados são mais específicos e precisos com concordância mais assertivas entre examinadores. Esse tipo de exame exige uma curva de aprendizado menor do avaliador, porém não há perda de eficácia no diagnóstico entre as duas modalidades (ZANINOTTO G, et al. 2019).

A manometria de alta resolução possibilitou estratificar com mais nitidez os graus da doença, pela Classificação de Chicago e impactando de forma significativa na escolha da terapêutica que será oferecida ao paciente. A análise do corpo esofágico permitiu classificar a doença em três variantes pela classificação de Chicago, tendo grande utilidade na definição prognóstica dos pacientes (LAFRAIA FM, 2017). Durante a investigação diagnóstica da paciente, foi realizada a manometria convencional após os achados típicos encontrados no estudo radiológico contrastado. Sendo evidenciado alterações compatíveis com acalasia vigorosa, que no caso específico foi caracterizado por ausência completa de peristalse durante todo corpo esofágico permitindo firmar o diagnóstico da patologia e propor a intervenção terapêutica a ser realizada.

No quesito tratamento, nenhuma terapia pode restaurar a funcionalidade dos músculos em um esôfago desnervado. A toxina botulínica deve ser usada em pacientes que possuem contraindicação para procedimentos mais invasivos e outros procedimentos como dilatação pneumática graduada, miotomia endoscópica por via oral e miotomia laparoscópica de Heller com cirurgia anti-refluxo possuem eficácia semelhante. A decisão sobre o tipo de tratamento deve ser baseada na idade do paciente e características, o tipo de acalasia pela manometria de alta resolução, a preferência do paciente e a experiência do centro (RONDÓN-CARVAJAL J, et.al., 2020).

As técnicas minimamente invasivas se apresentam com a vantagem de transcorrer em menor tempo operatório, promover menor perda sanguínea, menor dor no pós-operatório, menor índice de complicações pleuropulmonares, bem como redução do tempo de internação e recuperação precoce, quando em comparação a esofagectomia com toracotomia e laparotomia convencionais (COLA CB, et al., 2017). 
A cardiomiotomia à Heller modificada, por via videolaparoscópica, com realização de fundoplicatura acessória, é o principal procedimento cirúrgico para tratamento da acalasia, escolhido especialmente em pacientes jovens. É o método mais usado cirurgicamente, apresentando resultados satisfatórios em longo prazo e com reduzida morbimortalidade (MAGRI JÚNIOR JE, et al., 2017). No caso em questão, optou-se pela cardiomiotomia a Heller, associada a valvuloplastia antirreflexo a Toupet, por possibilidade de refluxo gastro-esofágico importante já que as fibras musculares do esfíncter inferior são seccionadas como parte da técnica operatória.

Na prática, boa parte dos cirurgiões faz uso de abordagem inicial menos agressiva, especialmente em pacientes mais jovens, como no caso acima descrito, em decorrência da elevada morbimortalidade associada à esofagectomia. Optar por uma abordagem mais conservadora e menos invasiva, como a laparoscópica, tem demonstrado ótimos resultados quanto à resolutividade e menores taxas de morbimortalidade pósoperatória (HERBELLA FA, 2015). Tal conduta pode postergar ou mesmo excluir a necessidade de realização da esofagectomia (ATES F, VAEZI MF, 2015).

Aproximadamente $5 \%$ dos pacientes que apresentam a doença irão para o estágio terminal ou megaesôfago, exigindo abordagem cirúrgica mais agressiva como a esofagectomia (AQUINO J, 2015). Esta se torna um desafio para cirurgião visto que com o progredir da patologia a configuração anatômica do órgão fica modificada e consequente o intra-operatório mais desafiador (RONDÓN-CARVAJAL J, et.al., 2020).

Diante de todo exposto, concluímos que a Acalasia por ser um distúrbio motor com sintomas gerais semelhantes com vários outros distúrbios do aparelho digestivo, a investigação diagnóstica precisa ser completa, com exames específicos, e com alto grau de suspeição clínica, visto que se observa na maioria das vezes diagnósticos tardios submetendo pacientes a longas maratonas de exames até a elucidação do quadro, comprometendo a abordagem terapêutica precoce, que se modifica conforme o grau da patologia, privando de certa forma os pacientes aos ganhos em qualidade de vida que o resultado do tratamento adequado da patologia pode oferecer. Neste contexto, observamos a importância de divulgação de relatos de casos para cada vez mais o diagnóstico se manter presente em nossa prática clínica.

De acordo com as literaturas analisadas, pode-se concluir que a indicação da abordagem cirúrgica nos casos de acalasia consiste em uma escolha criteriosa, sendo necessária uma análise crítica da experiência do serviço de cirurgia, das opções terapêuticas disponíveis e, principalmente, das condições clínicas do paciente em questão, visto que alguns procedimentos apresentam maiores taxas de morbimortalidade em decorrência de suas possíveis complicações. Dessa forma, o desenvolvimento de novos estudos analisando as abordagens e procedimentos mais recentes para correção da acalasia, mesmo sendo uma doença de rara incidência, é de fundamental importância, uma vez que ela pode atingir pessoas de todas as idades no mundo.

\section{REFERÊNCIAS}

1. ANDOLFI C, FISICHELLA PM. Meta-analysis of clinical outcome after treatment for achalasia based on manometric subtypes. British Journal of Surgery, 2019, 106(4): 332-341.

2. ATES F, VAEZI MF. The Pathogenesis and Management of Achalasia: Current Status and Future Directions. Gut Liver, 2015; 9(4): 449-63.

3. AQUINO J, et al. Surgical treatment analysis of idiopathic esophageal achalasia. ABCD Arq Bras Cir Dig., 2015; 28(2): 98-101.

4. COLA CB, et al. Esofagectomia vídeo-tóraco-laparoscópica com tempo torácico em posição pronada. Revista do Colégio Brasileiro de Cirurgiões, 2017; 44(5): 428-434.

5. HERBELLA FA, PATTI MG. Laparoscopic Heller myotomy and fundoplication in patients with end-stage achalasia. World J Surg, 2015; 39(7): 1631-3.

6. LAFRAIA FM, et al. A pictorial presentation of esophageal high resolution manometry current parameters. Arq Bras Cir Dig, 2017; 30(1): 69-71.

7. LOPES GA, et al. Acalasia de esôfago idiopática: Relato de caso e revisão da literatura. Brazilian Journal of Health Review, 2020; 3(5): 12267-12275.

8. MAGRI JÚNIOR JE, et al. Tratamento cirúrgico de acalasia pela realização de esofagocardiomiotomia com fundoplicatura videolaparoscópica à Heller-Pinotti em paciente com megaesôfago grau IV: Relato de caso. Rev. méd. Minas Gerais, 2017, 27: 1-4. 
9. MONTIEL-ROA AJ, et al. Surgical management of the esophageal achalasia in the IPS Central Hospital from january 2016 to december 2018. Cirugía paraguaya, 2020; 44(1): 16-18.

10. NETO RML, et al. Avaliação diagnóstica da acalasia do esôfago: dos sintomas à classificação de chicago. ABCD Arq Bras Cir Dig, 2018; 31(2): 1376.

11. SANTOS BC, et al. Gastroenterologia para o Estudante de Medicina. Liga Acadêmica de Gastroenterologia e Hepatologia da UFMG, 2021; 1(1): 27-34.

12. SCHLOTTMANN F, et al. Acalasia de esôfago: fisiopatologia, apresentação clínica e avaliação diagnóstica. The American Surgeon, 2018; 84(4): 467-472.

13. SWANSTRÖM LL. Acalasia: tratamento, situação atual e avanços futuros. Korean J Intern Med, 2019 ; 34 (6): $1173-$ 1180.

14. RONDÓN-CARVAJAL J, et al. Megaesophagus as a complication of achalasia: Case report and narrative literature review. Rev Colomb Gastroenterol. 2020; 35(4): 551-557.

15. ZANINOTTO G, et al. Management of acalasia em 2019. Opinião atual em gastroenterologia, 2019; 35(4): 356-362. 